\title{
Bounds on Shannon distinguishability in terms of partitioned measures
}

\author{
Alexey E. Rastegin \\ A. E. Rastegin, Department of Theoretical Physics, \\ Irkutsk State University, Gagarin Bv. 20, Irkutsk 664003, Russia
}

\begin{abstract}
A family of quantum measures like the Shannon distinguishability is presented. These measures are defined over the two classes of POVM measurements and related to separate parts in the expression for mutual information. Changes of Ky Fan's norms and the partitioned trace distances under the operation of partial trace are discussed. Upper and lower bounds on the introduced quantities are obtained in terms of partitioned trace distances and Uhlmann's partial fidelities. These inequalities provide a kind of generalization of the well-known bounds on the Shannon distinguishability. The notion of cryptographic exponential indistinguishability for quantum states is revisited. When exponentially fast convergence is required, all the metrics induced by unitarily invariant norms are shown to be equivalent.
\end{abstract}

PACS numbers: 03.67.-a, 03.65.Ta, 02.10.Ud

Keywords: Trace norm, Mutual information, Ky Fan's maximum principle, Partial fidelity, Exponential indistinguishability

\section{INTRODUCTION}

The advances of last decades have already shown a potential power of quantum systems as tools for information processing. In all the topics we deal with information by encoding symbols into quantum states. Any decoding procedure is done by quantum measurement at some stage. Because the outcomes of quantum measurement are not deterministic inevitably, convenient criteria of distinguishability for measurement statistics are of great importance. Classical probability distributions as well as mixed quantum states can be compared in many different ways 1 [3]. In the cryptographic context, the Shannon distinguishability has been found to be very valuable [1]. For example, security of quantum key distribution against wide classes of attacks has been stated with use of just this measure [4, 5].

In general, numerous approaches to measuring informational content of quantum states and their closeness have been developed [3]. Some of these measures are related to frequently used norms on the state space []. the trace norm distance is basic in many issues of quantum information. However, this measure is not monotone under taking tensor powers of density operators. Such a monotonicity is provided by the quantum fidelity elaborated by Uhlmann [7]. In Refs. [8, 9] a physical meaning of the fidelity was developed. Though both the trace distance and the fidelity are most important, more specialized measures can be needed with respect to the subject. For certain applications, some of them are more appropriate than others. So, the sub-fidelity [10] and the super-fidelity [10, 11] have been proposed as those measures that are easier to compute. In effect, many useful relations between various distances are known [1, 3]. Further, the Shannon distinguishability, the trace distance and the fidelity can be found to be equivalent in posing the exponential indistinguishability of protocols families [1]. Thus, studies of distinguishability measures and relations between them are still an actual issue of quantum information theory. The aim of the present work is to obtain more detailed characterization for Shannon distinguishability in a refined scale.

\section{NOTATION AND BACKGROUND}

In this section, we recall the notion of Shannon distinguishability as well as needed facts from matrix analysis. Let $B$ and $X$ be two random variables assigned to the input and output of a communication channel. Their probability distributions $p(b)$ and $p(x)$ are marginal with respect to the joint probability distribution $p(b, x)$, i.e.

$$
p(b)=\sum_{x \in X} p(b, x), \quad p(x)=\sum_{b \in B} p(b, x) .
$$

The relation $p_{b}(x) p(b)=p(b, x)=p_{x}(b) p(x)$ gives the conditional probabilities $p_{b}(x)$ and $p_{x}(b)$. In terms of the Shannon entropy, the mutual information is defined as

$$
I(B ; X) \triangleq H(B)+H(X)-H(B, X),
$$

where the joint entropy $H(B, X)=-\sum p(b, x) \log p(b, x)$ and logarithms are taken to base two. The measure (2.2) quantifies how the joint distribution $p(b, x)$ differs from the product of marginal distributions [3]. If we define the 
entropy of $B$ conditional on knowing $X$,

$$
H(B \mid X)=-\sum_{b \in B, x \in X} p(b, x) \log p_{x}(b),
$$

and also the conditional entropy of $X$ similarly, then $I(B ; X)=H(B)-H(B \mid X)=H(X)-H(X \mid B)[\underline{3}]$. So, the mutual information expresses the decrease of uncertainty through the detection, when uncertainty is quantified by the Shannon entropy. It is handy to use the binary entropy function $h(p) \equiv-p \log p-(1-p) \log (1-p)$ and the function $J(p) \equiv 1-h(p)$. For a binary input $B$ with equal prior probabilities [1, 4],

$$
I(B ; X)=\sum_{x \in X} p(x) J\left(p_{x}(0)\right)=\sum_{x \in X} p(x) J\left(p_{x}(1)\right),
$$

where $2 p(x)=p_{0}(x)+p_{1}(x), p_{x}(0)=p_{0}(x) /(2 p(x))$ and $p_{x}(1)=p_{1}(x) /(2 p(x))$. So, distinguishing the input is reduced to distinguishing $p_{0}(x)$ and $p_{1}(x)$ [1, 4]. Thinking of the expression (2.4) as a function of the two probability distributions $p_{0}(x)$ and $p_{1}(x)$, we define the Shannon distinguishability between them as

$$
S D\left(p_{0}(x), p_{1}(x)\right) \triangleq I(B ; X)=\sum_{x \in X} p(x) J\left(p_{x}(0)\right) .
$$

It is symmetric in the arguments, nonnegative and bounded from above by the inequality $S D\left(p_{0}(x), p_{1}(x)\right) \leq$ $D\left(p_{0}(x), p_{1}(x)\right) \equiv(1 / 2) \sum_{x \in X}\left|p_{0}(x)-p_{1}(x)\right|$.

A general quantum measurement is described by "positive operator-valued measure". The POVM $\mathcal{A}=\left\{\mathrm{A}_{x}\right\}(x \in X)$ is a set of positive matrices obeying $\sum_{x \in X} \mathrm{~A}_{x}=\mathbb{I}$, where $\mathbb{I}$ is the identity in $d$-dimensional Hilbert space $\mathcal{H}[3]$. When the property $\mathrm{A}_{x} \mathrm{~A}_{y}=\delta_{x y} \mathrm{~A}_{x}$ additionally holds, we have a standard measurement described by "projector-valued measure" (PVM). Applying the POVM $\mathcal{A}=\left\{\mathrm{A}_{x}\right\}$ to a system in the state $\rho_{i}$ results in the probability distribution $p_{i}^{\mathcal{A}}(x)=\operatorname{Tr}\left(\rho_{i} \mathrm{~A}_{x}\right)$. The quantity

$$
\mathrm{SD}^{\mathcal{A}}\left(\rho_{0}, \rho_{1}\right) \triangleq S D\left(p_{0}^{\mathcal{A}}(x), p_{1}^{\mathcal{A}}(x)\right)
$$

shows a distinguishability of the equiprobable states $\rho_{0}$ and $\rho_{1}$ once a particular POVM is used. The Shannon distinguishability between the two density matrices is then defined by [1, 4]

$$
\mathrm{SD}\left(\rho_{0}, \rho_{1}\right) \triangleq \sup \left\{\mathrm{SD}^{\mathcal{A}}\left(\rho_{0}, \rho_{1}\right): \mathcal{A} \in \mathrm{POVMs}\right\},
$$

where the supremum is taken over all POVMs. This quantity expresses the amount of information gained in performing a measurement. No analytic formula for $\operatorname{SD}\left(\rho_{0}, \rho_{1}\right)$ solely in terms of $\rho_{0}$ and $\rho_{1}$ is known [1]. It is for this reason that easily computable bounds are desired, particularly in cryptographic applications [4, 5]. Let $\widetilde{\rho}_{0}$ and $\widetilde{\rho}_{1}$ be two density operators defined on the tensor product $\mathcal{G} \otimes \mathcal{H}$. The Shannon distinguishability cannot increase under operation of partial trace, that is [4]

$$
\operatorname{SD}\left(\rho_{0}, \rho_{1}\right) \leq \operatorname{SD}\left(\widetilde{\rho}_{0}, \widetilde{\rho}_{1}\right),
$$

where the reduced operators $\rho_{i}=\operatorname{Tr}_{\mathcal{G}}\left(\widetilde{\rho}_{i}\right)$ are obtained by tracing-out $N$-dimensional space $\mathcal{G}$. The second upper bound is very important. Let $|\mathrm{A}|$ denote a unique positive square root of $\mathrm{A}^{\dagger} \mathrm{A}$. For any two density operators $\rho_{0}$ and $\rho_{1}$, there holds [1]

$$
\mathrm{SD}\left(\rho_{0}, \rho_{1}\right) \leq \frac{1}{2} \operatorname{Tr}\left|\rho_{0}-\rho_{1}\right| \equiv \mathrm{D}_{\mathrm{tr}}\left(\rho_{0}, \rho_{1}\right) .
$$

The upper bounds (2.8) and (2.9) are regularly used in analysis of vulnerability of quantum key distribution [4, 5]. We will also use both the lower bounds in terms of the quantum fidelity and the probability of error. The fidelity between density matrices $\rho_{0}$ and $\rho_{1}$ is defined as $\mathrm{F}\left(\rho_{0}, \rho_{1}\right)=\operatorname{Tr}\left|\sqrt{\rho_{0}} \sqrt{\rho_{1}}\right| \underline{3},[6]$. The probability of error between two probability distributions is given by $P E\left(p_{0}(x), p_{1}(x)\right) \equiv(1 / 2) \sum_{x \in X} \min \left\{p_{0}(x), p_{1}(x)\right\}$ [1]. Minimizing $P E\left(p_{0}^{\mathcal{A}}(x), p_{1}^{\mathcal{A}}(x)\right)$ over all measurements, the probability of error between $\rho_{0}$ and $\rho_{1}$ is obtained. This task occurs in the problem of state discrimination [19]. For two equiprobable states, we have [1]

$$
\operatorname{PE}\left(\rho_{0}, \rho_{1}\right)=\frac{1}{2}\left(1-\mathrm{D}_{\mathrm{tr}}\left(\rho_{0}, \rho_{1}\right)\right) .
$$

This value is actually reached by a PVM. The lower bounds on the Shannon distinguishability are then expressed as [1]

$$
\begin{aligned}
& 1-\mathrm{F}_{0}\left(\rho_{0}, \rho_{1}\right) \leq \mathrm{SD}\left(\rho_{0}, \rho_{1}\right), \\
& J\left(\operatorname{PE}\left(\rho_{0}, \rho_{1}\right)\right) \leq \operatorname{SD}\left(\rho_{0}, \rho_{1}\right) .
\end{aligned}
$$


Below some results of linear algebra will be needed. A unitarily invariant norm, in signs $\||\cdot|\|$, is a norm on square matrices that enjoys $\||A\|=\| U \mathrm{UV}\|\mid\|$ for any $\mathrm{A}$ and all unitary $\mathrm{U}, \mathrm{V}$ [12]. Two classes of such norms are specially important. For real $q \geq 1$, the Schatten $q$-norm of operator A on $\mathcal{H}$ is defined by $\|\mathrm{A}\|_{q}=\left(\sum_{x=1}^{d} s_{x}(\mathrm{~A})^{q}\right)^{1 / q}$ [6, 12], where the singular values $s_{x}(\mathrm{~A})$ are eigenvalues of $|\mathrm{A}|$. This class includes the trace norm $\|\mathrm{A}\|_{\mathrm{tr}}$ for $q=1$, the Frobenius norm $\|\mathrm{A}\|_{F}$ for $q=2$, and the spectral norm $\|\mathrm{A}\|_{\infty}$ for $q \rightarrow \infty[6]$. The Schatten norms have found use in various questions of quantum information theory [13]. For $k=1, \ldots, d$, the Ky Fan $k$-norm $\|\mathrm{A}\|_{(k)}$ is defined as the sum of $k$ largest singular values [12]. We obtain the spectral norm for $k=1$ and the trace norm for $k=d$. We will also use Ky Fan's maximum principle [14] which can be expressed as follows. If the eigenvalues $\lambda_{x}$ of Hermitian operator $\mathrm{A}$ are so arranged that $\lambda_{1} \geq \lambda_{2} \geq \cdots \geq \lambda_{d}$, then

$$
\sum_{x=1}^{k} \lambda_{x}=\max \{\operatorname{Tr}(\Pi \mathrm{A}): \mathbf{0} \leq \Pi \leq \mathbb{I}, \operatorname{Tr}(\Pi)=k\},
$$

where the maximization is over positive matrices $\Pi$ with trace $k$ that satisfy $\Pi \leq \mathbb{I}$.

\section{DEFINITIONS OF PARTITIONED MEASURES}

In this section, the definitions of new distinguishability measures are given. For obtaining a more thorough description, separate terms in the entry for mutual information should be estimated. That is, we are interested in weight of separate components in the right-hand side of (2.5). This can be attained by consideration of partial sums under the decreasing order of summands. Let $\#(X)$ denote the cardinality of the set $X$.

Definition III.1. The $k$-th partial Shannon distinguishability between two the probability distributions $p_{0}(x)$ and $p_{1}(x)$ is defined by

$$
S D_{k}\left(p_{0}(x), p_{1}(x)\right) \triangleq \max \left\{\sum_{x \in Y} p(x) J\left(p_{x}(0)\right): Y \subset X, \#(Y)=k\right\} .
$$

We have $S D_{l}\left(p_{0}(x), p_{1}(x)\right) \leq S D_{k}\left(p_{0}(x), p_{1}(x)\right)$ whenever $l \leq k$, and the entry $\#(Y)=k$ can be replaced by $\#(Y) \leq k$. For the two probability distributions, we obtain a family of \# $(X)$ nonnegative symmetric measures which are all bounded. Let us proceed to the case of quantum system with the state space $\mathcal{H}$. For given POVM $\mathcal{A}, k$-th partial Shannon distinguishability between $\rho_{0}$ and $\rho_{1}$ is naturally put as

$$
\operatorname{SD}_{k}^{\mathcal{A}}\left(\rho_{0}, \rho_{1}\right)=S D_{k}\left(p_{0}^{\mathcal{A}}(x), p_{1}^{\mathcal{A}}(x)\right) .
$$

Further, it is not insignificant that a family of utilized measurements may be constrained in some ways. Restrictions can be due to used apparatus, applied protocol or strategy, and perhaps a priori information on the signal quantum states. So, it is of some interest to consider specialized classes of POVM measurements.

Definition III.2. Let $\mathfrak{S}$ be a family of POVMs. Then the $k$-th partial Shannon distinguishability with respect to $\mathfrak{S}$ is defined by

$$
\operatorname{SD}_{k}^{\mathfrak{S}}\left(\rho_{0}, \rho_{1}\right) \triangleq \sup \left\{\operatorname{SD}_{k}^{\mathcal{A}}\left(\rho_{0}, \rho_{1}\right): \mathcal{A} \in \mathfrak{S}\right\}
$$

In the following, we will consider the two important families of POVMs. Putting $d=\operatorname{dim}(\mathcal{H})$, the first family $\mathfrak{A}$ is defined as

$$
\mathfrak{A} \triangleq\left\{\mathcal{A}: \operatorname{Tr}\left(\mathrm{A}_{x}\right) \leq 1 \forall x \in X, \#(X) \leq d^{2}\right\} .
$$

Indispensable one-rank POVMs are all contained in this family. As a rule, quantum information tasks lead to hard problems of nonlinear optimization. Due to famous Davies' results [15], an analysis can often be simplified to a POVM with one-rank elements whose number is limited by $d \leq \#(X) \leq d^{2}$. Using this fact, Fuchs and Peres have shown that the optimal detection for a two-state system is reached with a two-dimensional eavesdropper's probe [16]. Such POVMs are sufficient for optimal unambiguous discrimination [17] which is widely adopted in quantum key distribution [18]. We also know that POVMs with elements of higher rank can never give more mutual information than maximizing one-rank POVM. So, the family $\mathfrak{A}$ of measurements is of importance. The second family $\mathfrak{B}$ is defined as

$$
\mathfrak{B} \triangleq\left\{\mathcal{A}: \operatorname{Tr}\left(\mathrm{A}_{x}\right) \geq 1 \forall x \in X\right\}
$$


In this definition, we have $\#(X) \leq d$ with necessity. The family $\mathfrak{B}$ contains all the projective measurements which are easier to realize experimentally. Moreover, in discrimination between two quantum states the average probability of error is minimized by POVM that is actually a PVM [19].

In addition, reasons for using the families $\mathfrak{A}$ and $\mathfrak{B}$ are connected with interpretations of the partitioned trace distances and the partial fidelities in terms of measurement statistics. Such relations between classical distinguishability measures and their quantum versions are used in various contexts [3, 9]. The $k$-th partitioned trace distance between $\rho_{0}$ and $\rho_{1}$ is expressed by $[20]$

$$
\mathrm{D}_{k}\left(\rho_{0}, \rho_{1}\right)=\frac{1}{2}\left\|\rho_{0}-\rho_{1}\right\|_{(k)}
$$

For $k=d$, this definition leads to the trace norm distance $\mathrm{D}_{\mathrm{tr}}\left(\rho_{0}, \rho_{1}\right)$ which can also be put via extremal properties of quantum operations [21]. The partitioned distances enjoy many properties of the trace norm distance, including the unitary invariance and the strong convexity [20]. The derivation of these results is essentially based on the Ky Fan maximum principle (2.13). Let us put the $k$-th classical trace distance between probability distributions $p_{0}(x)$ and $p_{1}(x)$ as

$$
D_{k}\left(p_{0}(x), p_{1}(x)\right) \triangleq \max \left\{\frac{1}{2} \sum_{x \in Y}\left|p_{0}(x)-p_{1}(x)\right|: Y \subset X, \#(Y)=k\right\} .
$$

A kind of statistical interpretation is then expressed by

$$
\mathrm{D}_{k}\left(\rho_{0}, \rho_{1}\right)=\max \left\{D_{k}\left(p_{0}^{\mathcal{A}}(x), p_{1}^{\mathcal{A}}(x)\right): \mathcal{A} \in \mathfrak{A}\right\}
$$

where the maximum is actually reached by the one-rank PVM [20].

In Ref. [22], Uhlmann introduced $k$-th partial fidelity as

$$
\mathrm{F}_{k}\left(\rho_{0}, \rho_{1}\right)=\min \left\{\sum_{x \in Y} s_{x}\left(\sqrt{\rho_{0}} \sqrt{\rho_{1}}\right): \#(Y)=d-k\right\} .
$$

These quantities allow to resolve the equivalence of pairs of mixed states under invertible transformations [22]. The partial fidelities enjoy the joint concavity [22] and other useful properties [23]. For $k=0$, we have the regular quantum fidelity $\mathrm{F}_{0}\left(\rho_{0}, \rho_{1}\right)=\operatorname{Tr}\left|\sqrt{\rho_{0}} \sqrt{\rho_{1}}\right|$. Note that $\mathrm{F}_{k}\left(\rho_{0}, \rho_{1}\right) \equiv \mathrm{F}_{0}\left(\rho_{0}, \rho_{1}\right)-\left\|\sqrt{\rho_{0}} \sqrt{\rho_{1}}\right\|_{(k)}$ in terms of the Ky Fan $k$-norm. We also define the $k$-th fidelity between probability distributions as [23]

$$
F_{k}\left(p_{0}(x), p_{1}(x)\right) \triangleq \min \left\{\sum_{x \in Y} \sqrt{p_{0}(x) p_{1}(x)}: Y \subset X, \#(Y)=\#(X)-k\right\} .
$$

A kind of statistical interpretation of the partial fidelities is provided by [23]

$$
\mathrm{F}_{k}\left(\rho_{0}, \rho_{1}\right) \leq \inf \left\{F_{k}\left(p_{0}^{\mathcal{A}}(x), p_{1}^{\mathcal{A}}(x)\right): \mathcal{A} \in \mathfrak{B}\right\}
$$

where the equality cannot always be reached in general.

\section{CHANGES UNDER THE OPERATION OF PARTIAL TRACE}

In quantum theory, the state of a subsystem of a composite quantum system is described by a reduced density operator. Except for the opaque method, for attack on a quantum cryptosystem the intruder entangles his probes with transmitted carriers somehow 24]. In either case, the intruder finally deals with those density matrices that are results of the operation of partial trace. Hence we are interested in how used quantitative measures may be changed by this operation. Let $\widetilde{\rho}_{0}$ and $\widetilde{\rho}_{1}$ be density matrices on $\mathcal{G} \otimes \mathcal{H}$ and $\rho_{i}=\operatorname{Tr}_{\mathcal{G}}\left(\widetilde{\rho}_{i}\right)$. For the partial fidelities there holds [23]

$$
\mathrm{F}_{k}\left(\rho_{0}, \rho_{1}\right) \geq \mathrm{F}_{(N k)}\left(\widetilde{\rho}_{0}, \widetilde{\rho}_{1}\right)
$$

We shall now give a similar relation for partitioned trace distances. For distances between the marginal probability distributions $p_{i}(x)=\sum_{1 \leq \xi \leq N} \widetilde{p}_{i}(\xi, x)$, we have

$$
D_{k}\left(p_{0}(x), p_{1}(x)\right)=\sum_{x \in Y^{\prime}}\left|\sum_{\xi=1}^{N} \widetilde{p}_{0}(\xi, x)-\sum_{\xi=1}^{N} \widetilde{p}_{1}(\xi, x)\right| \leq D_{(k N)}\left(\widetilde{p}_{0}(\xi, x), \widetilde{p}_{1}(\xi, x)\right)
$$


due to the triangle inequality. Here $Y^{\prime}$ denotes a $k$-subset of $X$ such that the maximum in (3.7) is reached. Using the statistical interpretation (3.8), we could obtain a quantum version of (4.2). However, it is of some interest to consider more general question about the Ky Fan norms. In Ref. [25] the problem is resolved for those unitarily invariant norms that are multiplicative over tensor products. In particular, there hold

$$
\|\mathrm{A}\|_{F} \leq \sqrt{N}\|\widetilde{\mathrm{A}}\|_{F}, \quad\|\mathrm{~A}\|_{\infty} \leq N\|\widetilde{\mathrm{A}}\|_{\infty}
$$

where $\mathrm{A}$ is taken from $\widetilde{\mathrm{A}}$ by the operation of partial trace over $N$-dimensional space. Except for $k=1$, however, Ky Fan's norms are not multiplicative in this way [25]. So the following result is valuable.

Lemma IV.1. For each operator $\widetilde{\mathrm{A}}$ on the tensor product $\mathcal{G} \otimes \mathcal{H}, \operatorname{dim}(\mathcal{G})=N$, and its partial trace $\mathrm{A}=\operatorname{Tr}_{\mathcal{G}}(\widetilde{\mathrm{A}})$, there holds

$$
\|\mathrm{A}\|_{(k)} \leq\|\widetilde{\mathrm{A}}\|_{(N k)}
$$

Proof. (a) Let $\widetilde{A}$ be Hermitian. If $\widetilde{A}=\widetilde{B}-\widetilde{C}$ is the Jordan decomposition, then both $B \equiv \operatorname{Tr}_{\mathcal{G}}(\widetilde{B}), C \equiv \operatorname{Tr}_{\mathcal{G}}(\widetilde{C})$ are positive (but not mutually orthogonal in general) and $A=B-C$. Since $A$ is also Hermitian, there holds $\|\mathrm{A}\|_{(k)}=\operatorname{Tr}[(\mathrm{P}-\mathrm{Q}) \mathrm{A}]$ for some mutually orthogonal projectors $\mathrm{P}$ and $\mathrm{Q}$ that satisfy $\operatorname{rank}(\mathrm{P}+\mathrm{Q}) \leq k[20]$. Due to positivity of $P, Q, B$, and $C$,

$$
\begin{aligned}
\|\mathrm{A}\|_{(k)} & =\operatorname{Tr}_{\mathcal{H}}[(\mathrm{P}-\mathrm{Q}) \mathrm{A}]=\operatorname{Tr}_{\mathcal{H}}[(\mathrm{P}-\mathrm{Q})(\mathrm{B}-\mathrm{C})] \\
& \leq \operatorname{Tr}_{\mathcal{H}}[(\mathrm{P}+\mathrm{Q})(\mathrm{B}+\mathrm{C})]=\operatorname{Tr}_{\mathcal{G} \otimes \mathcal{H}}\left[\left(\mathbb{I}_{\mathcal{G}} \otimes \Pi\right)(\widetilde{B}+\widetilde{\mathrm{C}})\right],
\end{aligned}
$$

where we used $\Pi=P+Q$ and the properties of the trace including [6]

$$
\operatorname{Tr}_{\mathcal{H}}(\Pi \mathrm{B})=\operatorname{Tr}_{\mathcal{G} \otimes \mathcal{H}}\left[\left(\mathbb{I}_{\mathcal{G}} \otimes \Pi\right) \widetilde{\mathrm{B}}\right]
$$

Since $\widetilde{B}+\widetilde{C}=|\widetilde{A}|$ by definition, its eigenvalues are positive and $\operatorname{rank}\left(\mathbb{I}_{\mathcal{G}} \otimes \Pi\right) \leq N k$, Ky Fan's maximum principle (2.13) provides the relation (4.4) for the Hermitian case.

$(\mathrm{b})^{1}$ For arbitrary $\widetilde{\mathrm{A}}$, we define its left absolute value $|\widetilde{\mathrm{A}}|_{L}=\left(\widetilde{\mathrm{A}} \widetilde{\mathrm{A}}^{\dagger}\right)^{1 / 2}$ and right absolute value $|\widetilde{\mathrm{A}}|_{R}=\left(\widetilde{\mathrm{A}}{ }^{\dagger} \widetilde{\mathrm{A}}\right)^{1 / 2}$. It follows from Hermiticity of these two operators and part (a) that the Ky Fan $(N k)$-norm satisfies

$$
\|\widetilde{\mathrm{A}}\|_{(N k)}=\left\||\widetilde{\mathrm{A}}|_{L}\right\|_{(N k)}=\left\||\widetilde{\mathrm{A}}|_{R}\right\|_{(N k)} \geq \max \left\{\left\|\mathrm{A}_{L}\right\|_{(k)},\left\|\mathrm{A}_{R}\right\|_{(k)}\right\},
$$

where $A_{L} \equiv \operatorname{Tr}_{\mathcal{G}}\left(|\widetilde{A}|_{L}\right)$ and $A_{R} \equiv \operatorname{Tr}_{\mathcal{G}}\left(|\widetilde{A}|_{R}\right)$. We claim that the right-hand side of (4.7) is not less than $\|A\|_{(k)}$. Using the singular value decomposition $\widetilde{A}=\widetilde{U} \widetilde{D} \widetilde{V}$, we obtain $|\widetilde{A}|_{L}=\widetilde{U} \widetilde{D} \widetilde{U}^{\dagger}$ and $|\widetilde{A}|_{R}=\widetilde{V^{\dagger}} \widetilde{D} \widetilde{V}$. To prove the claim, we write

$$
\widetilde{\mathrm{U}}=\sum_{i, j=1}^{N}|i\rangle\left\langle j\left|\otimes \mathrm{U}_{i j}, \quad \widetilde{\mathrm{D}}=\sum_{j=1}^{N}\right| j\right\rangle\left\langle j\left|\otimes \mathrm{D}_{j j}, \quad \widetilde{\mathrm{V}}=\sum_{i, j=1}^{N}\right| i\right\rangle\langle j| \otimes \mathrm{V}_{i j}
$$

with respect to an orthonormal basis $\{|i\rangle\}$ in the space $\mathcal{G}$. That is, $\widetilde{U}$ may be viewed as a $N$-by- $N$ block matrix with blocks $\mathrm{U}_{i j}$, and so on. By calculations, we get

$$
\mathrm{A}=\sum_{i, j=1}^{N} \mathrm{U}_{i j} \mathrm{D}_{j j} \mathrm{~V}_{j i}, \quad \mathrm{~A}_{L}=\sum_{i, j=1}^{N} \mathrm{U}_{i j} \mathrm{D}_{j j} \mathrm{U}_{j i}^{\dagger}, \quad \mathrm{A}_{R}=\sum_{i, j=1}^{N} \mathrm{~V}_{i j}^{\dagger} \mathrm{D}_{j j} \mathrm{~V}_{j i}
$$

because $\operatorname{Tr}_{\mathcal{G}}(|i\rangle\langle j| \otimes \Theta)=\langle j \mid i\rangle \Theta$ for any operator $\Theta$ on $\mathcal{H}$. Let us use the two 1 -by- $N^{2}$ block matrices

$$
\begin{aligned}
& \mathrm{L}=\left[\begin{array}{llll}
\mathrm{U}_{11} \sqrt{\mathrm{D}}_{11} & \mathrm{U}_{12}{\sqrt{\mathrm{D}_{22}}} & \ldots & \mathrm{U}_{N N} \sqrt{\mathrm{D}}_{N N}
\end{array}\right], \\
& \mathrm{R}=\left[\begin{array}{llll}
\mathrm{V}_{11}^{\dagger} \sqrt{\mathrm{D}}_{11} & \mathrm{~V}_{12}^{\dagger}{\sqrt{\mathrm{D}_{22}}} & \ldots & \mathrm{V}_{N N}^{\dagger} \sqrt{\mathrm{D}}_{N N}
\end{array}\right] .
\end{aligned}
$$

\footnotetext{
${ }^{1}$ An extension to the non-Hermitian case was noted by one of the referees.
} 
It is easy to check that $A=L R^{\dagger}, A_{L}=L^{\dagger}$ and $A_{R}=R^{\dagger}$. We finally have

$$
\left\|\mathrm{LR}^{\dagger}\right\|_{(k)} \leq\left(\left\|\mathrm{LL}^{\dagger}\right\|_{(k)}\left\|\mathrm{RR}^{\dagger}\right\|_{(k)}\right)^{1 / 2} \leq \max \left\{\left\|\mathrm{LL}^{\dagger}\right\|_{(k)},\left\|\mathrm{RR}^{\dagger}\right\|_{(k)}\right\} .
$$

The inequality on the left is a Cauchy-Schwarz inequality for ordinary products of rectangular matrices (of the same size) and given unitarily invariant norms (see, e.g., the inequality (3.5.22) in [26]).

In particular, we have $\|\mathrm{A}\|_{\operatorname{tr}} \leq\|\widetilde{\mathrm{A}}\|_{\operatorname{tr}}$ for $k=d,\|\mathrm{~A}\|_{\infty} \leq\|\widetilde{\mathrm{A}}\|_{(N)}$ for $k=1$. The latter relation is stronger than the second inequality of (4.3) (except when the largest singular value of $\widetilde{\mathrm{A}}$ has multiplicity $\geq N$ ). The partitioned trace distances satisfy

$$
\mathrm{D}_{k}\left(\rho_{0}, \rho_{1}\right) \leq \mathrm{D}_{(N k)}\left(\widetilde{\rho}_{0}, \widetilde{\rho}_{1}\right) .
$$

As it is well known, the trace norm distance cannot increase and the fidelity cannot decrease under the partial trace. This endorses the mind reason that objects become less distinguishable when only partial information is available. All the partitioned distances enjoy the mentioned property in the sense of relations (4.2) and (4.13). For Uhlmann's partial fidelities, the relation (4.1) is useful in this regard.

\section{BASIC INEQUALITIES}

Because the Shannon distinguishability measures $\operatorname{SD}_{k}^{\mathfrak{S}}\left(\rho_{0}, \rho_{1}\right)$ are positive-valued, we are foremost interested in upper bounds similar to (2.8) and (2.9). First, we present the inequalities with the partitioned trace distances. Corresponding bounds for density operators are essentially based on the relations for probability distributions.

Theorem V.1. Let the measures $S D_{k}, D_{k}$, and $F_{k}$ be defined by formulas (3.1), 3.7), and (3.10) respectively. For any two probability distributions and $k=0,1, \ldots, \#(X)$,

$$
S D_{k}\left(p_{0}(x), p_{1}(x)\right) \leq D_{k}\left(p_{0}(x), p_{1}(x)\right) \leq 1-F_{k}\left(p_{0}(x), p_{1}(x)\right) .
$$

Proof. Firstly, we denote by $Y^{\prime}$ a $k$-subset of $X$ such that

$$
S D_{k}\left(p_{0}(x), p_{1}(x)\right)=\sum_{x \in Y^{\prime}} p(x) J\left(p_{x}(0)\right) .
$$

As it is shown in [1, 4] , for $0 \leq r \leq 1$ there holds $J(r) \leq|2 r-1|$, whence

$$
S D_{k}\left(p_{0}(x), p_{1}(x)\right) \leq \sum_{x \in Y^{\prime}} p(x)\left|\left(p_{0}(x) / p(x)\right)-1\right|=\frac{1}{2} \sum_{x \in Y^{\prime}}\left|p_{0}(x)-p_{1}(x)\right|
$$

due to $p(x)=\left(p_{0}(x)+p_{1}(x)\right) / 2$. By (3.7), the right-hand side of (5.2) does not exceed $k$-th partitioned distance $D_{k}\left(p_{0}(x), p_{1}(x)\right)$. Secondly, let $Y \subset X$ be a $k$-subset such that the maximum in (3.7) is reached. Because $\sum_{x \in X} p_{i}(x)=1$, we write

$$
\begin{aligned}
2 D_{k}\left(p_{0}(x), p_{1}(x)\right) & \leq \sum_{x \in Y}\left(p_{0}(x)+p_{1}(x)\right)=2-\sum_{x \in Y_{c}}\left(p_{0}(x)+p_{1}(x)\right) \\
& \leq 2-\sum_{x \in Y_{c}} 2 \sqrt{p_{0}(x) p_{1}(x)} \leq 2-2 F_{k}\left(p_{0}(x), p_{1}(x)\right),
\end{aligned}
$$

where $Y_{c}$ is the complement of $Y$ and, therefore, $\#\left(Y_{c}\right)=\#(X)-k$.

Due to (3.8) and (5.1), for all $\mathcal{A} \in \mathfrak{A}$ we have $\operatorname{SD}_{k}^{\mathcal{A}}\left(\rho_{0}, \rho_{1}\right) \leq \mathrm{D}_{k}\left(\rho_{0}, \rho_{1}\right)$. If each number of the set is not greater than $\mathrm{D}_{k}\left(\rho_{0}, \rho_{1}\right)$ then the supremum of the set does also obey this. Combining the claim with (4.13), we obtain an extension of the upper bounds (2.8) and (2.9) in terms of partitioned measures.

Theorem V.2. Let the measures $\mathrm{SD}_{k}^{\mathfrak{A}}$ and $\mathrm{D}_{k}$ be defined by formulas (3.3), for the family (3.4), and (3.6) respectively. For any two density matrices and $k=0,1, \ldots, d$,

$$
\mathrm{SD}_{k}^{\mathfrak{A}}\left(\rho_{0}, \rho_{1}\right) \leq \mathrm{D}_{k}\left(\rho_{0}, \rho_{1}\right) .
$$

If operators $\rho_{0}$ and $\rho_{1}$ are taken as $\rho_{i}=\operatorname{Tr}_{\mathcal{G}}\left(\widetilde{\rho}_{i}\right)$ over $N$-dimensional space $\mathcal{G}$ then

$$
\operatorname{SD}_{k}^{\mathfrak{A}}\left(\rho_{0}, \rho_{1}\right) \leq \mathrm{D}_{(k N)}\left(\widetilde{\rho}_{0}, \widetilde{\rho}_{1}\right) .
$$


The inequality (5.4) generalizes the well-known bound (2.9) to the case considered. In analysis of quantum information protocols, the operation of partial trace is inevitable. Apparently, no simple version of (2.8) exists for partial measures $\operatorname{SD}_{k}^{\mathfrak{A}}\left(\rho_{0}, \rho_{1}\right)$. But the bound (2.8) is rather useful in a ready combination with (2.9), namely

$$
\mathrm{SD}\left(\rho_{0}, \rho_{1}\right) \leq \mathrm{D}_{\operatorname{tr}}\left(\widetilde{\rho}_{0}, \widetilde{\rho}_{1}\right)
$$

Indeed, the Shannon distinguishability itself is typically unknown. For instance, in a study of security problem the result (5.6) is actually used [4, 5]. So, a useful analog of (5.6) is provided by (5.5). Let us proceed to the relations with the partial fidelities.

Theorem V.3. Let the measures $\mathrm{SD}_{k}^{\mathfrak{B}}$ and $\mathrm{F}_{k}$ be defined by formulas (3.3), for the family (3.5), and (3.9) respectively. For any two density matrices and $k=0,1, \ldots, d$,

$$
\operatorname{SD}_{k}^{\mathfrak{B}}\left(\rho_{0}, \rho_{1}\right) \leq 1-\mathrm{F}_{k}\left(\rho_{0}, \rho_{1}\right)
$$

If operators $\rho_{0}$ and $\rho_{1}$ are taken as $\rho_{i}=\operatorname{Tr}_{\mathcal{G}}\left(\widetilde{\rho}_{i}\right)$ over $N$-dimensional space $\mathcal{G}$ then

$$
\operatorname{SD}_{k}^{\mathfrak{B}}\left(\rho_{0}, \rho_{1}\right) \leq \operatorname{SD}_{k}^{\widetilde{\mathfrak{B}}}\left(\widetilde{\rho}_{0}, \widetilde{\rho}_{1}\right)
$$

Proof. By (5.1) and (3.11), we get $\operatorname{SD}_{k}^{\mathcal{A}}\left(\rho_{0}, \rho_{1}\right) \leq 1-\mathrm{F}_{k}\left(\rho_{0}, \rho_{1}\right)$ for any POVM $\mathcal{A} \in \mathfrak{B}$. Combining this with the definition (3.3) at once gives (5.7). Further, the set $\widetilde{\mathcal{A}}=\left\{\mathbb{I}_{\mathcal{G}} \otimes \mathrm{A}_{x}\right\}$ is a POVM on the total space $\mathcal{G} \otimes \mathcal{H}$ and generates the probabilities

$$
\operatorname{Tr}_{\mathcal{H}}\left(\rho_{i} \mathrm{~A}_{x}\right)=\operatorname{Tr}_{\mathcal{G} \otimes \mathcal{H}}\left[\widetilde{\rho}_{i}\left(\mathbb{I}_{\mathcal{G}} \otimes \mathrm{A}_{x}\right)\right]
$$

whence $\operatorname{SD}_{k}^{\mathcal{A}}\left(\rho_{0}, \rho_{1}\right)=\operatorname{SD}_{k}^{\widetilde{\mathcal{A}}}\left(\widetilde{\rho}_{0}, \widetilde{\rho}_{1}\right)$. The fact $\operatorname{Tr}_{\mathcal{G} \otimes \mathcal{H}}\left(\mathbb{I}_{\mathcal{G}} \otimes \mathrm{A}_{x}\right) \geq N$ implies $\widetilde{\mathcal{A}} \in \widetilde{\mathfrak{B}}$. So the left-hand side of $(\underline{5.8})$ is the supremum over a certain subfamily of $\widetilde{\mathfrak{B}}$.

Note that the combined relation $\operatorname{SD}_{k}^{\mathfrak{B}}\left(\rho_{0}, \rho_{1}\right) \leq 1-\mathrm{F}_{k}\left(\widetilde{\rho}_{0}, \widetilde{\rho}_{1}\right)$ may rather be suitable in calculations. Both the basic inequalities (5.4) and (5.7) can be posed as majorization relations. Notions of majorization theory are very useful, for instance, in matrix analysis [12] and studies of quantum systems [27]. Let $q=\left(q_{1}, \ldots, q_{m}\right)$ and $r=\left(r_{1}, \ldots, r_{m}\right)$ be elements of real space $\mathbb{R}^{m}$. We say that $q$ is weakly submajorized by $r$, in symbols $q \prec_{w} r$, when [12]

$$
\sum_{x=1}^{k} q_{x}^{\downarrow} \leq \sum_{x=1}^{k} r_{x}^{\downarrow}, \quad 1 \leq k \leq m
$$

where the arrows down indicate that vector coordinates are put in decreasing order. Denoting $p_{i}^{\mathcal{A}}(x)=\operatorname{Tr}\left(\rho_{i} \mathrm{~A}_{x}\right)$ and $J_{x}^{\mathcal{A}}=J\left(p_{x}^{\mathcal{A}}(0)\right) \equiv J\left(p_{x}^{\mathcal{A}}(1)\right)$, the inequalities (5.4) and (5.7) are merely reformulated as

$$
\begin{aligned}
& \left(p_{0}^{\mathcal{A}}+p_{1}^{\mathcal{A}}\right) J^{\mathcal{A}} \prec_{\boldsymbol{w}} s\left(\rho_{0}-\rho_{1}\right) \quad \forall \mathcal{A} \in \mathfrak{A}, \\
& \left(p_{0}^{\mathcal{A}}+p_{1}^{\mathcal{A}}\right) J^{\mathcal{A}} \prec_{\boldsymbol{w}} 2 s\left(\sqrt{\rho_{0}} \sqrt{\rho_{1}}\right) \quad \forall \mathcal{A} \in \mathfrak{B},
\end{aligned}
$$

where $p_{x}^{\mathcal{A}}(i)=p_{i}^{\mathcal{A}}(x) /\left(p_{0}^{\mathcal{A}}(x)+p_{1}^{\mathcal{A}}(x)\right)$ and the definitions (3.6) and (3.9) are expanded. The majorization relations (5.11) and (5.12) give another description for components of Shannon distinguishability measures in more detailed terms. In a certain sense, these statements are complementary to each other, since they are related to the two different families of practically important POVM measurements. The following bounds are analogs of (2.11) and (2.12) for the partial Shannon distinguishability measures.

Theorem V.4. Let the measures $\mathrm{SD}_{k}^{\mathfrak{A}}$ and $\mathrm{SD}_{k}^{\mathfrak{B}}$ be defined by (3.3), for the classes (3.4) and (3.5), $\mathrm{F}_{0}$ by (3.9) and $\mathrm{PE}$ by (2.10). For $k=0,1, \ldots, d$, there hold

$$
\begin{aligned}
\frac{k}{d^{2}}\left(1-\mathrm{F}_{0}\left(\rho_{0}, \rho_{1}\right)\right) & \leq \operatorname{SD}_{k}^{\mathfrak{A}}\left(\rho_{0}, \rho_{1}\right), \\
\frac{k}{d} J\left(\operatorname{PE}\left(\rho_{0}, \rho_{1}\right)\right) & \leq \operatorname{SD}_{k}^{\mathfrak{B}}\left(\rho_{0}, \rho_{1}\right) .
\end{aligned}
$$

Proof. If we put the partial sums $Q_{k}=\sum_{x=1}^{k} q_{x}^{\downarrow}$, then (see lemma 3 in [23])

$$
m Q_{k} \geq k Q_{m}, \quad k=0,1, \ldots, m \text {. }
$$


Due to this relation and the condition $\#(X) \leq d^{2}$ in (3.4), for each $\mathcal{A} \in \mathfrak{A}$ we have

$$
\operatorname{SD}_{k}^{\mathcal{A}}\left(\rho_{0}, \rho_{1}\right) \geq \frac{k}{d^{2}} \operatorname{SD}^{\mathcal{A}}\left(\rho_{0}, \rho_{1}\right) .
$$

Hence the suprema of the two sides of (5.16) satisfy $\operatorname{SD}_{k}^{\mathfrak{A}}\left(\rho_{0}, \rho_{1}\right) \geq\left(k / d^{2}\right) \mathrm{SD}^{\mathfrak{A}}\left(\rho_{0}, \rho_{1}\right)$. The measure $\operatorname{SD}^{\mathfrak{A}}\left(\rho_{0}, \rho_{1}\right)$ is the Shannon distinguishability (2.7) itself, as the family $\mathfrak{A}$ certainly contains one-rank POVM that optimizes the mutual information. So the bound (5.13) follows from (2.11). Second, let $\Pi \in \mathfrak{B}$ be PVM such that $\operatorname{PE}\left(\rho_{0}, \rho_{1}\right)=$ $P E\left(p_{0}^{\Pi}(x), p_{1}^{\Pi}(x)\right)$. Using (5.15) and the definitions (3.3) and (3.5), we have

$$
\operatorname{SD}_{k}^{\mathfrak{B}}\left(\rho_{0}, \rho_{1}\right) \geq \mathrm{SD}_{k}^{\Pi}\left(\rho_{0}, \rho_{1}\right) \geq \frac{k}{d} \mathrm{SD}^{\Pi}\left(\rho_{0}, \rho_{1}\right)
$$

From the relation $S D\left(p_{0}^{\Pi}(x), p_{1}^{\Pi}(x)\right) \geq J\left(P E\left(p_{0}^{\Pi}(x), p_{1}^{\Pi}(x)\right)\right)$, which is known for probability distributions [1], we obtain (5.14).

The significance of Theorems V.2 $\mathrm{V.3}$ and $\mathrm{V} .4$ is that, while the quantum Shannon distinguishability measures are unknown in a closed form, the inequalities provide a useful way to estimate them. A more detailed characterization is given with respect to those POVMs that are important from the practical viewpoint. Both the partitioned trace distances and partial fidelities enjoy a kind of statistical interpretation. On the other hand, they do not have a direct information-theoretic meaning. Such a treatment may be expressed via the partial varieties of Shannon distinguishability. Due to the lower bounds (5.13) and (5.14), partitioned measures can also be applied in the context of exponential indistinguishability.

\section{NOTES ON EXPONENTIAL INDISTINGUISHABILITY}

Comparing the protocol implementation (i.e. the family of protocols) with the ideal protocol specification, we would like that the probability of cheating for each participant vanishes exponentially, as taken security parameter $n$ increases [1]. This label may sign the length of a string, the number of rounds, or the number of carriers transmitted. Let $\left\{X_{0}\right\}=\left\{X_{0}^{(1)}, X_{0}^{(2)}, X_{0}^{(3)}, \ldots\right\}$ and $\left\{X_{1}\right\}=\left\{X_{1}^{(1)}, X_{1}^{(2)}, X_{1}^{(3)}, \ldots\right\}$ be families of random variables with the probability distributions $\left\{p_{0}^{(1)}, p_{0}^{(2)}, p_{0}^{(3)}, \ldots\right\}$ and $\left\{p_{1}^{(1)}, p_{1}^{(2)}, p_{1}^{(3)}, \ldots\right\}$. These families are exponentially indistinguishable if there exist some $n_{0}$ and $\varepsilon \in(0 ; 1)$ such that [1]

$$
D\left(p_{0}^{(n)}, p_{1}^{(n)}\right) \leq \varepsilon^{n} \quad \forall n \geq n_{0} .
$$

The motivation and examples are presented in [1]. The measures $D\left(p_{0}, p_{1}\right), P E\left(p_{0}, p_{1}\right), F_{0}\left(p_{0}, p_{1}\right)$ and $S D\left(p_{0}, p_{1}\right)$ are found to be equivalent when we require exponentially fast convergence to the values that are obtained for two identical distributions (i.e., $D=0, P E=1 / 2, F_{0}=1$, and $S D=0$ ). It is natural to take two families $\left\{\rho_{0}^{(n)}\right\}=$ $\left\{\rho_{0}^{(1)}, \rho_{0}^{(2)}, \rho_{0}^{(3)}, \ldots\right\}$ and $\left\{\rho_{1}^{(n)}\right\}=\left\{\rho_{1}^{(1)}, \rho_{1}^{(2)}, \rho_{1}^{(3)}, \ldots\right\}$ of density operators on $d$-dimensional space $\mathcal{H}$. The two families are exponentially indistinguishable if there exist some $n_{0}$ and $\varepsilon \in(0 ; 1)$ such that 1$]$

$$
\mathrm{D}_{\operatorname{tr}}\left(\rho_{0}^{(n)}, \rho_{1}^{(n)}\right) \leq \varepsilon^{n} \quad \forall n \geq n_{0} .
$$

It is valuable that an equivalence of similar kind takes place in the quantum case. Namely, an exponentially fast convergence with respect to one of the measures $\mathrm{D}, \mathrm{PE}, \mathrm{F}_{0}$ and $\mathrm{SD}$ implies the same with respect to all these measures [1]. Below, we will analyze a convergence with respect to both the partitioned trace distances and partial varieties of Shannon distinguishability.

Theorem VI.1. Let $\left\{\mathrm{M}_{k}\right\}$ be one of three measure series $\left\{\mathrm{D}_{k}\right\}_{k=1}^{d},\left\{\mathrm{SD}_{k}^{\mathfrak{A}}\right\}_{k=1}^{d^{2}}$, and $\left\{\mathrm{SD}_{k}^{\mathfrak{B}}\right\}_{k=1}^{d}$ defined by formulas (3.6) and (3.3), for the classes (3.4) and (3.5), respectively. If families $\left\{\rho_{0}^{(n)}\right\}$ and $\left\{\rho_{1}^{(n)}\right\}$ are exponentially indistinguishable with respect to measure $\mathrm{M}_{k_{0}}$ of series $\left\{\mathrm{M}_{k}\right\}$ then they are exponentially indistinguishable with respect to all measures of the series.

Proof. (a) Suppose the families $\left\{\rho_{0}^{(n)}\right\}$ and $\left\{\rho_{1}^{(n)}\right\}$ are exponentially indistinguishable with respect to $\mathrm{D}_{k_{0}}(1 \leq$ $\left.k_{0} \leq d\right)$. So there exist integer $n_{0}$ and real $\varepsilon \in(0 ; 1)$ such that

$$
\mathrm{D}_{k_{0}}\left(\rho_{0}^{(n)}, \rho_{1}^{(n)}\right) \leq \varepsilon^{n} \quad \forall n \geq n_{0} .
$$


Due to (5.15), the trace norm distance obeys $\mathrm{D}_{\operatorname{tr}}\left(\rho_{0}^{(n)}, \rho_{1}^{(n)}\right) \leq\left(d / k_{0}\right) \varepsilon^{n}$, whenever $n \geq n_{0}$. Let $n_{\varepsilon}$ denote the smallest integer such that

$$
\left(\frac{d}{k_{0}}\right)^{1 / n_{\varepsilon}} \cdot \varepsilon=\epsilon<1
$$

This value clearly exists because $\varepsilon<1$ and $\left(d / k_{0}\right)^{1 / n} \rightarrow 1$ in the limit $n \rightarrow \infty$. By calculations, we get

$$
n_{\varepsilon}=\left\lfloor\frac{\ln \left(d / k_{0}\right)}{-\ln \varepsilon}\right\rfloor+1 \text {. }
$$

For all $n \geq \max \left\{n_{0}, n_{\varepsilon}\right\}$, we then obtain $\mathrm{D}_{\operatorname{tr}}\left(\rho_{0}^{(n)}, \rho_{1}^{(n)}\right) \leq \epsilon^{n}$. By definition, each partitioned trace distance is not larger than the trace norm distance. This completes the proof for the series $\left\{\mathrm{D}_{k}\right\}_{k=1}^{d}$. (b) It follows from (5.16) and related reasons that

$$
\mathrm{SD}\left(\rho_{0}^{(n)}, \rho_{1}^{(n)}\right) \leq \frac{d^{2}}{k_{0}} \operatorname{SD}_{k_{0}}^{\mathfrak{A}}\left(\rho_{0}^{(n)}, \rho_{1}^{(n)}\right) \leq \frac{d^{2}}{k_{0}} \varepsilon^{n}
$$

for given $k_{0}$ and all $n \geq n_{0}$. By the above arguments, for all $n \geq \max \left\{n_{0}, n_{*}\right\}$ we have

$$
\mathrm{SD}\left(\rho_{0}^{(n)}, \rho_{1}^{(n)}\right) \leq \epsilon_{*}{ }^{n},
$$

where $\epsilon_{*}<1$ and $n_{*}$ are defined by replacing $d$ with $d^{2}$ in the formulas (6.4) and (6.5). By definition, each measure $\mathrm{SD}_{k}^{\mathfrak{A}}$ does not exceed the total sum $\mathrm{SD}^{\mathfrak{A}}$ and, therefore, the left-hand side of (6.7). (c) Suppose that for given $k_{0}$ and all $n \geq n_{0}$

$$
\mathrm{SD}_{k_{0}}^{\mathfrak{B}}\left(\rho_{0}^{(n)}, \rho_{1}^{(n)}\right) \leq \varepsilon^{n} .
$$

Using (5.14) and the above reasons, there holds $J\left(\operatorname{PE}\left(\rho_{0}^{(n)}, \rho_{1}^{(n)}\right)\right) \leq \epsilon^{n}$, whenever $n \geq \max \left\{n_{0}, n_{\varepsilon}\right\}$. By calculus, for $r \in[0 ; 1]$ we get $J(r) \geq(2 / \ln 2)(r-1 / 2)^{2}$, whence

$$
\frac{1}{2}-\operatorname{PE}\left(\rho_{0}^{(n)}, \rho_{1}^{(n)}\right) \leq \sqrt{\frac{\ln 2}{2}}(\sqrt{\epsilon})^{n} .
$$

This implies exponentially fast convergence with respect to the probability of error and, therefore [1], with respect to the Shannon distinguishability itself. The latter is not less than the measure $\mathrm{SD}^{\mathfrak{B}} \geq \mathrm{SD}_{k}^{\mathfrak{B}}$, where $k=1, \ldots, d$.

Thus, an equivalence stated in the paper [1] really is much more broad in character. Indeed, exponentially indistinguishable families of density operators enjoy this property with respect to all the above partitioned measures (except for the partial fidelities). With respect to the question of interest, some measures may be easier to calculate or experimentally estimate. So, a freedom in formulation of exponential indistinguishability is useful. It turns out that such a treatment can be proceeded to each metric induced by a unitarily invariant norm. Due to the Fan dominance theorem (see, e.g., Corollary (3.5.9) in [26]), many relations with Ky Fan's norms can be extended to all unitarily invariant norms. For any traceless Hermitian operator A, there hold [28]

$$
\|\mathrm{A}\|_{\infty} \leq\|\| \mathrm{Z}\left|\left\|^{-1}\right\|\right| \mathrm{A}\|\|, \quad\|\mathrm{A}\| \mid \leq \frac{\|\mathrm{Z} \mid\|}{2}\|\mathrm{~A}\|_{\text {tr }},
$$

where $\mathbf{Z}=\operatorname{diag}(1,1,0, \ldots, 0)$. Note that the multiplier of $\|\mathbf{A}\|$ tr in (6.10) is independent of $\mathbf{A}$. We say that $\left\{\rho_{0}^{(n)}\right\}$ and $\left\{\rho_{1}^{(n)}\right\}$ are exponentially indistinguishable with respect to the induced metric if there exist some $m_{0}$ and $\delta \in(0 ; 1)$ such that

$$
\left\|\left|\rho_{0}^{(n)}-\rho_{1}^{(n)} \|\right| \leq \delta^{n} \quad \forall n \geq m_{0} .\right.
$$

Since the difference between two density matrices is traceless, we can use (6.10). For given unitarily invariant norm, the value of ||$Z|| \mid$ is a fixed positive number. For the Schatten $q$-norm, say, ||$|Z|||=2^{1 / q}$. The claimed equivalence can be observed in the same manner, as the statement of Theorem VI.1 has been proved. Using the first inequality of (6.10), the formula (6.11) leads to exponentially fast convergence with respect to the metric $2 \mathrm{D}_{1}$ induced by the spectral norm. By Theorem VI.1, the exponentially fast convergence takes place with respect to all measures of the series $\left\{\mathrm{D}_{k}\right\}_{k=1}^{d}$ including the trace norm distance. That is, any convergence of a kind (6.11) implies the convergence of a kind (6.2). Conversely, the second inequality of (6.10) and the formula (6.2) lead to $\left\|\left|\rho_{0}^{(n)}-\rho_{1}^{(n)}\right|\right\||\leq \|||Z||| \varepsilon^{n}$, whenever $n \geq n_{0}$. By some technical work, this implies that the inequality (6.11) holds for each unitarily invariant norm. 
Theorem VI.2. If two families of density matrices are exponentially indistinguishable with respect to one metric induced by a unitarily invariant norm then these families are exponentially indistinguishable with respect to all the metrics induced by unitarily invariant norms.

Thus, unitarily invariant norms provide flexible tools for analysis of distinguishability including the cryptographic context. Some of them are very well studied, for instance, the spectral norm and the trace norm. So their nice properties are widely adopted in many respects. However, induced metrics do not have a direct information-theoretic content. This sense is rather a feature of the Shannon distinguishability and its partial varieties because they are defined via the mutual information. But closed analytical expressions for them are not known. Hence all the above relations between different measures are important. Moreover, quantum exponential indistinguishability can be resolved by means of any metric induced by a unitarily invariant norm.

\section{CONCLUSION}

The partial Shannon distinguishability measures have been presented. A more detailed characterization is given with respect to both the adopted measurements and separate terms in the sum for mutual information. Since the operation of partial trace is typical, a special issue of Ky Fan's norms after the partial trace was firstly resolved by the statement of Lemma IV.1. In general, the optimizing measurement can be unknown or infeasible with an available equipment. So the studied quantities are relevant when the optimal POVM is replaced by a POVM from the classes considered. The upper bounds on the introduced measures are given in a form of simple inequalities using the partitioned trace distances (see Theorem $\overline{\nabla .2}$ ) and Uhlmann's partial fidelities (see Theorem $\overline{V .3}$ ). In Theorem V.4 the relevant lower bounds are also presented. Theorem VI.1 treats the proposed measures in the context of exponentially indistinguishable families of quantum states. For such two families, a distinguisher may be unable to identify the source of a given sample, even if he is not restricted to polynomial-time calculations. In the case of exponentially fast convergence, all the metrics induced by unitarily invariant norms are shown to be tantamount (see Theorem VI.2). This equivalence is expected to be useful in designing indistinguishable families of density matrices.

\section{Acknowledgments}

The comments of anonymous referees were very valuable. I am particularly grateful for pointing out part (b) of the proof of Lemma IV.1.

[1] Fuchs, C.A., van de Graaf, J.: Cryptographic distinguishability measures for quantum mechanical states. IEEE Trans. Inf. Theory 45, 1216-1227 (1999)

[2] Gilchrist, A., Langford, N.K., Nielsen, M.A.: Distance measures to compare real and ideal quantum processes. Phys. Rev. A 71, $062310(2005)$

[3] Hayashi, M.: Quantum Information: An Introduction. Springer, Berlin (2006)

[4] Biham, E., Boyer, M., Brassard, G., van de Graaf, J., Mor, T.: Security of quantum key distribution against all collective attacks. Algorithmica 34, 372-388 (2002)

[5] Biham, E., Boyer, M., Boykin, P.O., Mor, T., Roychowdhury, V.: A proof of the security of quantum key distribution. J. Cryptology 19, 381-439 (2006)

[6] Watrous, J.: CS 798: Theory of quantum information. University of Waterloo, http://www.cs.uwaterloo.ca/ watrous/quant-info/lecture-notes/all-lectures.pdf (2008)

[7] Uhlmann, A.: The transition probability in the state space of a *algebra. Rep. Math. Phys. 9, 273-279 (1976)

[8] Jozsa, R.: Fidelity for mixed quantum states. J. Mod. Opt. 41, 2315-2323 (1994)

[9] Fuchs, C.A., Caves, C.M.: Mathematical techniques for quantum communication theory. Open Syst. Inf. Dyn. 3, 345-356 (1995)

[10] Miszczak, J.A., Puchała, Z., Horodecki, P., Uhlmann, A., Życzkowski, K.: Sub- and super-fidelity as bounds for quantum fidelity. Quantum Inf. Comput. 9, 0103-0130 (2009)

[11] Mendonça, P.E.M.F., Napolitano, R.d.J., Marchiolli, M.A., Foster, C.J., Liang, Y.-C.: Alternative fidelity measure between quantum states. Phys. Rev. A 78, 052330 (2008)

[12] Bhatia, R.:Matrix Analysis. Springer, New York (1997)

[13] Watrous, J.: Notes on super-operator norms induced by Schatten norms. Quantum Inf. Comput. 5, 58-68 (2005)

[14] Fan, K.: On a theorem of Weyl concerning eigenvalues of linear transformations. I. Proc. Nat. Acad. Sci. USA 35, 652-655 (1949) 
[15] Davies, E.B.: Information and quantum measurement. IEEE Trans. Inf. Theory 24, 596-599 (1978)

[16] Fuchs, C.A., Peres, A.: Quantum-state disturbance versus information gain: Uncertainty relations for quantum information. Phys. Rev. A 53, 2038-2045 (1996)

[17] Peres, A., Terno, D.R.: Optimal distinction between non-orthogonal quantum states. J. Phys. A: Math. Gen. 31, 7105-7111 (1998)

[18] Brandt, H.E.: Unambiguous state discrimination in quantum key distribution. Quantum Inf. Process. 4, 387-398 (2005)

[19] Helstrom, C.W.: Quantum Detection and Estimation Theory. Academic Press, New York (1976)

[20] Rastegin, A.E.: Partitioned trace distances. Quantum Inf. Process. 9, 61-73 (2010)

[21] Rastegin, A.E.: Trace distance from the viewpoint of quantum operation techniques. J. Phys. A: Math. Theor. 40, 9533-9549 (2007)

[22] Uhlmann, A.: On "partial" fidelities. Rep. Math. Phys. 45, 407-418 (2000)

[23] Rastegin, A.E.: Some properties of partial fidelities. Quantum Inf. Comput. 9, 1069-1080 (2009)

[24] Brandt, H.E.: Optimum probe parameters for entangling probe in quantum key distribution. Quantum Inf. Process. 2, 37-79 (2003)

[25] Lidar, D.A., Zanardi, P., Khodjasteh, K.: Distance bounds on quantum dynamics. Phys. Rev. A 78, 012308 (2008)

[26] Horn, R.A. and Johnson, C.R.: Topics in Matrix Analysis. Cambridge University Press, Cambridge (1991)

[27] Nielsen, M.A., Vidal, G.: Majorization and the interconversion of bipartite states. Quantum Inf. Comput. 1, 76-93 (2001)

[28] Audenaert, K.M.R., Eisert, J.: Continuity bounds on the quantum relative entropy. J. Math. Phys. 46, 102104 (2005) 